\title{
Editorial
}

\section{Information, Knowledge and Wisdom}

On last September, in Fortaleza city, the XIII Brazilian Meeting on Inorganic Chemistry (BMIC) consolidated the great expectations from the preceding years, dealing successfully with the challenges imposed by the increasing complexity of the area, encompassing coordination chemistry, macrocyclic compounds, biomimetic systems, catalysis, metallodrugs, mixed-valence compounds, molecular magnets, theoretical modeling, spectroscopy, electrochemistry and photochemistry; and extending the frontiers to the nanoparticles, films and nanotechnology. This special number of the Journal of the Brazilian Chemical Society reflects such a richness of chemical content, also surely observed in many other areas. However, while such richness can be translated into development, it also conveys a critical concern, as expressed by the sequence: information $\rightarrow$ knowledge $\rightarrow$ wisdom. Such a progression is better represented by a pyramid supported on information, as the base, mediated by the knowledge, to reach wisdom, in the top. Since Science is being shaped by the increasing complexity of the knowledge, the solid construction of such pyramid becomes an important goal to be pursued. Nowadays, the information base is exponentially expanding, imposing a dramatic challenge for the students aiming a rapid insertion in their professional lives. For this reason, the search for information is becoming a major task in education, particularly at the undergraduate and graduate levels. However, some negative consequences are now appearing, as discussed in the VI Brazilian Workshop on Graduate Programs in Chemistry, in Maceio city, on last November. In this event, several reported cases by the participants raised serious concerns regarding the scientific background of the graduate students, who can exhibit an impressive list of publications, but a rather poor performance in terms of knowledge. Such a problem is probably much more complex, but one of the causes may be the strong emphasis on the information alone. As a matter of fact, stimulated by the increasing facilities of sharing and copying, especially from the internet, information is vigorously shaping our society. The risk, however, is to decline from the next steps of evolution, in which information should be converted into knowledge, through a prepared mind, and handled with creativity, for opening the access to wisdom. One can trace a similar parallel by extrapolating the knowledge pyramid to the production area, in searching for the best route of innovation. Information, as the base of the knowledge, can not be denied; however, its usefulness critically depends on our mind, on our ability of analysing and interpreting, in the same way as a data base will always require software for showing its meaning. A professional, lacking enough background and understanding, will not make the best use of information. In spite of this, the current emphasis on information is reaching a point in which the educational area is being invaded by the so called long distance courses, where the presence of the student in the classroom is not required. Therefore, the noble mission of the professor, as educator, is being buried under the myths of the information society. It already seems that many students are assuming their easy access to the information, effective enough to reach their goal, thus limiting their learning to the reading of the supporting materials and notes, while running away from the classroom, from the professor's influence and from the educational structure. This is particularly critical in experimental courses, such as in Chemistry, where the laboratory plays an essential role in the formation of the student. Without understanding, information is not enough to capture the enthusiasm from the students. Lacking interest and motivation, their absence in the classroom is just a terrible consequence, which should be treated on due time to prevent their abandon from the course. This is becoming a serious problem in our community. It is necessary to pursue the understanding, through the teaching of concepts and principles. Therefore, great efforts should be directed in order to surpass the information society and build up a real knowledge society, aiming at, one day, a society of wisdom.

Henrique E. Toma

(IQ-USP) 